\title{
Gastric intestinal metaplasia and helicobacter pylori testing
}

\begin{abstract}
Gastric intestinal metaplasia (GIM) is a frequent precancerous lesion and is detected in nearly 1 of every 4 patients undergoing upper endoscopy. Helicobacter pylori (H. pylori.) is believed to be the cause of majority of intestinal-type gastric adenocarcinoma by causing chronic active gastritis, which may evolve to atrophic gastritis, GIM, dysplasia, and cancer. Whether H. pylori eradication leads to reversal of GIM remains controversial, but several studies suggest some benefit. Since $H$. pylori exhibits tissue tropism, we believe that the progression to atrophic gastritis and intestinal metaplasia drives Helicobacter pylori out of the gastric mucosa. We hypothesized that gastric biopsy alone for the detection of H. pylori infection may be less accurate in the context of GIM, especially if biopsies fail to sample non-metaplastic tissue. Hence, another method of H. pylori testing like urease breath test (UBT) or serology must be performed to evaluate for H. pylori. A retrospective chart review was conducted using Electronic Medical Records (EMR) at Dayton Veteran's Affairs Medical Center. Our study showed that pathologic testing for H. pylori was completed in almost all cases of GIM and only a small proportion of patients had H. pylori identified from their biopsy. This is in accordance with our hypothesis that gastric biopsy has lower accuracy in the presence of IM because of inability of H.Pylori to grow on metaplastic tissue. H. pylori eradication could reduce the risk of further development of dysplasia and subsequent gastric malignancy. Therefore, we recommend that patients with GIM should undergo additional diagnostic tests if the initial biopsy is negative for H. pylori.
\end{abstract}

Keywords: gastric, intestinal, metaplasia, helicobacter pylori, testing, atrophic, precancerous, diagnosed, eradicated
Volume 4 Issue 6 - 2016

\author{
Drew Triplett DO, Thomas Murphy, Mumtaz \\ Khan Sajjad, Sangeeta Agrawal \\ Division of Gastroenterology,Wright State University, USA
}

\begin{abstract}
Correspondence: Sangeeta Agrawal, Associate Professor of Medicine, Division of Gastroenterology,Wright State University, Dayton VA Medical Center, 4100 West 3rd Street, Dayton, USA, Tel 9372694842, Fax 937-262-2154,

Email Sangeeta.agrawal@va.gov, sagrawalgi@gmail.com
\end{abstract}

Received: May 20, 2016 | Published: May 30, 2016
Abbreviations: GIM, gastric intestinal metaplasia; UBT, urease breath test; IRB, institutional review board; EMR, electronic medical records; IM, intestinal metaplasia

\section{Introduction}

Gastric intestinal metaplasia (GIM) is a relatively frequent precancerous lesion with a six to eleven fold increase in gastric cancer risk. ${ }^{1-4}$ GIM is thought to be in the cascade of gastric carcinogenesis coming after multifocal atrophic gastritis but before dysplasia. ${ }^{1,5-7}$ The prevalence of GIM depends upon geographical location and is higher in countries with high prevalence of $H$. pylori infection. ${ }^{3}$ In the United States the overall rate of abnormal stomach mucosa increases with age, $4.9 \%$ biopsies showing GIM in a large multi-center retrospective study. ${ }^{8}$ It is believed that the progression to atrophic gastritis and intestinal metaplasia drives $H$. pylori out of the gastric mucosa since the epithelium is no longer a suitable environment for $H$. pylori. ${ }^{9}$ Complete intestinal metaplasia does not allow $H$. pylori adherence. ${ }^{10}$ Previous studies of GIM positive biopsies have shown low rates of $H$. pylori infection. ${ }^{11}$ It is thought that $H$. pylori may be detected in other non-metaplastic areas of the gastric mucosa in the presence GIM if biopsies are taken from that area.

Recommendations have been previously made to determine $H$. pylori infection status by tissue-based tests and serology. ${ }^{12}$ Urease breath testing (UBT) or serologic testing has also been recommended as a way to diagnose $H$. pylori infection in patients with GIM whose histological staining for $H$. pylori is negative. ${ }^{13}$ Furthermore, infected patients should be treated to eradicate $H$. pylori. ${ }^{12,14}$ Eradication is thought to lessen the occurrence of gastric cancer by preventing progression of precancerous lesions. ${ }^{15,16}$ However, gastric cancer has been documented after eradication of $H$. pylori ${ }^{17}$ and some authors believe that once GIM is found there is no need to test for H. pylori. ${ }^{18}$ Reversibility of GIM is controversial, but some trials suggest a benefit. ${ }^{19-24}$ The Maastricht IV consensus report says there is no evidence that $\mathrm{H}$ pylori eradication can lead to regression of intestinal metaplasia, leaving one to conclude that there would be no benefit to testing for $H$. pylori in patients with GIM. ${ }^{25}$ However the report goes on to say, "the exact point of no return has not been identified" and gives a grade A recommendation to eradicate H. pylori for the prevention of gastric cancer in patients with severe atrophy. ${ }^{25}$ Metaanalyses in 2011 and 2014 suggest that $H$. pylori eradication correlates with improvement in GIM $^{26,27}$ while a 2007 meta-analysis showed reversibility of atrophic gastritis, but not GIM. ${ }^{28}$ These recent metaanalyses led one author to say "further prospective studies are needed to determine the optimal strategy about $H$. pylori eradication to prevent gastric cancer". ${ }^{29}$ Based on the hypothesis that some patients with GIM may benefit by having $H$. pylori infection diagnosed and then eradicated, we designed the retrospective chart review study to see what had been done in these cases at our institution over the last 10 years.

\section{Methods}

This retrospective chart review was conducted using Electronic medical records (EMR) at Dayton VA Medical Center (DVAMC). After Institutional review board (IRB) approval, a list of patients with a histological diagnosis of GIM for the last 10 years was obtained from the pathology department. Each case was reviewed individually and biopsies that were clearly labeled as "gastric" were included in this study. Any biopsies labeled "gastroesophageal junction" or "esophageal" were excluded from this study. The EMR 
of each patient with biopsy proven GIM was reviewed individually. Demographic data, endoscopy reports, biopsy reports and laboratory data was reviewed and documented.

\section{Results}

A total of 54 patients were identified with histological diagnosis of gastric intestinal metaplasia (GIM). The median age was 68 years, 51 (94.4\%) were male, $45(83.3 \%)$ were caucasian, 43 (79.6\%) were present or former smokers. Documentation of testing for $H$. pylori was found for all (100\%) patients with 12/54 (22.2\%) testing positive in pathology specimen. Of the remaining 42 patients with histological absence of $H$. pylori, serologic testing was done in 8/42 (19\%): 4/8 $(50 \%)$ tested positive, $2 / 8(25 \%)$ tested negative and $2 / 8(25 \%)$ were equivocal. No other diagnostic method is available in this hospital for $H$. pylori. There were 34/42 (81\%) patients with GIM and $H$. pylori negative biopsies who did not have serological testing done, but 2 of them had anti-H. pylori treatment given empirically and 2 had protocol biopsies done 2 years later. Both the patients who had equivocal results on serological testing had protocol biopsies done 1-2 years later. Only 14/54 (25.9\%) patients were treated for $\mathrm{H}$. pylori eradication after the diagnosis of GIM (Figure 1).

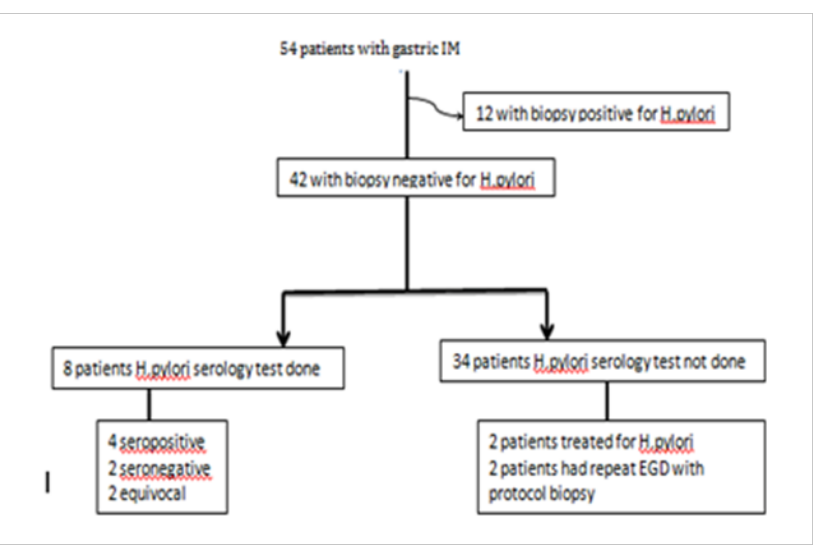

Figure I Flow diagram of patients included in the study.

IM, intestinal metaplasia

\section{Discussion}

Gastric intestinal metaplasia can be a precursor to gastric cancer caused by $H$. pylori infection. Histology is considered to be the "gold standard" test for H. pylori. ${ }^{30}$ However, if the metaplastic tissue is no longer a suitable host for $H$. pylori and it migrates to a more histological normal gastric tissue, then we may miss diagnosing some cases of $H$. pylori infection in patients with GIM. There is a difference in opinion among experts about whether $H$. pylori eradication is beneficial in patients with GIM. If $H$. pylori is eradicated and further pre-malignant cellular changes are prevented we believe that we should test for and eradicate H. pylori in patients with GIM. Which test is best to look for $H$. pylori infection in the setting of GIM and negative histology is a question that remains to be answered. In our institution H. pylori serology is the only other available test. Lower diagnostic accuracy of $H$. pylori with histologic examination has been described in the setting of GIM. ${ }^{12}$ Urea breath testing (UBT) in the setting of GIM and $H$. pylori negative biopsies has been studied retrospectively. UBT has decreased sensitivity if the patient is on proton pump inhibitor, therefore stool antigen test or serology should be considered as alternatives. ${ }^{12,13}$ Arguments have been made advocating for UBT as the most sensitive and specific test for $H$. pylori. ${ }^{31}$ We are not advocating the use of serology over UBT, just that further testing should be done in patients with IM and H. pylori negative biopsies.

Our study shows that pathologic testing for $H$. pylori was completed in almost all cases of GIM and only a small proportion $(22.2 \%)$ of patients had $H$. pylori identified from their biopsy. This is in accordance with our hypothesis that gastric biopsy has lower accuracy in the presence of GIM because of the inability of $H$. pylori to grow on intestinal metaplastic tissue. While only 8 out of 42 patients with negative biopsies had serological testing performed, 4 out of those $8(50 \%)$ had positive serology. It is unclear if this represents the whole population of our patients with biopsy proven GIM and negative H. pylori histologic staining. Larger studies from multiple centers are warranted. If we identified more patients with continued active infection, $H$. pylori eradication could reduce the risk of further development of dysplasia and subsequent gastric malignancy. Currently there is no evidence to support that GIM is reversible, but it is reasonable to suggest that if active $H$. pylori infection can be identified and treated, further cellular changes could be limited. Therefore, we recommend that patients with gastric intestinal metaplasia should undergo additional diagnostic tests for $H$. pylori if the biopsy is negative.

\section{Acknowledgment}

None.

\section{Conflicts of interset}

The author declares there is no conflict of interest.

\section{Funding}

None.

\section{References}

1. Correa P. Human gastric carcinogenesis: a multistep and multi factorial process: First American Cancer Society Award Lecture on Cancer Epidemiology and Prevention. Cancer Res. 1992;52(24):6735-6740.

2. Leung WK, Sung JJY. Review article; intestinal metaplasia and gastric carcinogenesis. Aliment Pharmacol Ther. 2002;16(7):1209-1216.

3. Zullo A, Hassan C, Romiti A, et al. Follow-up of intestinal metaplasia in the stomach: When, how and why. World $J$ Gastrointest Oncol. 2002;4(3):30-36.

4. Bleibel W, Frye J, Gomez J. Intestinal Metaplasia of the Stomach Is Associated with an Increased Risk of Gastric Cancer in a Western Population. Am J Gastroenterol. 2001;108:S49.

5. de Vries AC, Kuipers EJ. Epidemiology of premalignant gastric lesions: implications for the development of screening and surveillance strategies. Helicobacter. 2007;12(Suppl 2):22-31.

6. Correa P. A human model of gastric carcinogenesis. Cancer Res. 1998;48(13):3554-3560.

7. Correa P, Piazuelo MB. The gastric precancerous cascade. J Dig Dis. 2012;13(1):2-9.

8. Sonnenberg A, Genta RM. Changes in the Gastric Mucosa with Aging. Clin Gastroenterol Hepatol. 2015;13(13):2276-2281.

9. Kang HY, Kim N, Park YS, et al. Progression of atrophic gastritis and intestinal metaplasia drives Helicobacter pylori out of the gastric mucosa. Dig Dis Sci. 2006;51(12):2310-2315.

10. Smith VC, Genta RM. Role of Helicobacter pylori gastritis in gastric atrophy, intestinal metaplasia, and gastric neoplasia. Microsc Res Tech. 2000;48(6):313-320. 
11. Genta RM, Gürer IE, Graham DY, et al. Adherence of Helicobacter pylori to areas of incomplete intestinal metaplasia in the gastric mucosa. Gastroenterology. 1996;111(5):1206-1211.

12. Correa P, Piazuelo MB, Wilson KT. Pathology of gastric intestinal metaplasia: clinical implications. Am J Gastroenterol. 2010;105(3):493498.

13. Galiatsatos P, Wyse J, Szilagyi A. Accuracy of biopsies for Helicobacter pylori in the presence of intestinal metaplasia of the stomach. Turk $J$ Gastroenterol. 2014;25(1):19-23.

14. Galiatsatos P. Surveillance of Gastric Intestinal Metaplasia: Is It Warranted? Journal of Gastroenterology and Hepatology Research. 2014;3(12):1364-1366.

15. Kodama M, Murakami K, Okimoto $\mathrm{T}$, et al. Ten-year prospective follow-up of histological changes at five points on the gastric mucosa as recommended by the updated Sydney system after Helicobacter pylori eradication. J Gastroenterol. 2012;47(4):394-403.

16. Correa P, Fontham ET, Bravo JC, et al. Chemoprevention of gastric dysplasia: randomized trial of antioxidant supplements and antihelicobacter pylori therapy. J Natl Cancer Inst. 2002;92(23):1881-1888.

17. de Vries AC, Kuipers EJ, Rauws EA. Helicobacter pylori eradication and gastric cancer: when is the horse out of the barn? Am J Gastroenterol. 2009;104(6):1342-1345.

18. Ince AT. Utility if diagnosing $H$. pylori in presence of intestinal metaplasia. Turk J Gastroenterol. 2014;25(2):227-228.

19. Sung JJ, Lin SR, Ching JY, et al. Atrophy and intestinal metaplasia one year after cure of $H$. pylori infection: a prospective, randomized study. Gastroenterol. 2000;119(1):7-14.

20. Ito M, Haruma K, Kamada T, et al. Helicobacter pylori eradication therapy improves atrophic gastritis and intestinal metaplasia: a 5-year prospective study of patients with atrophic gastritis. Aliment Pharmacol. 2002;16(8):1449-1456.
21. Kokkola A, Sipponen P, Rautelin H, et al. The effect of Helicobacter pylori eradication on the natural course of atrophic gastritis with dysplasia. Aliment Pharmacol Ther. 2012;16(3):515-520.

22. Ohkusa T, Fujiki K, Takashimizu I, et al. Improvement in atrophic gastritis and intestinal metaplasia in patients in whom Helicobacter pylori was eradicated. Ann Intern Med. 2001;134(5):380-386.

23. Mera R, Fontham ET, Bravo LE, et al. Long term follow-up of patients treated for helicobacter pylori infection. Gut. 2005;54:1536-1540.

24. Kodama M, Murakami K, Okimoto T, et al. Helicobacter pylori eradication improves gastric atrophy and intestinal metaplasia in longterm observation. Digestion. 2012;85(2):126-130.

25. Malfertheiner P, Megraud F, O'Morain CA, et al. Management of Helicobacter pylori infection--the Maastricht IV/ Florence Consensus Report. Gut. 2012;61:646-664.

26. Wang J, Xu L, Shi R, et al. Gastric atrophy and intestinal metaplasia before and after Helicobacter pylori eradication: a meta-analysis. Digestion. 2011;83(4):253-260.

27. Kong YJ, Yi HG, Dai JC, et al. Histological changes of gastric mucosa after Helicobacter pylori eradication: a systematic review and metaanalysis. World J Gastroenterol. 2014;20(19):5903-5911.

28. Rokkas T, Pistiolas D, Sechopoulos P, et al. The long-term impact of Helicobacter pylori eradication on gastric histology: a systematic review and meta-analysis. Helicobacter. 2007;12(Suppl 2):32-38.

29. Park YH, Kim N. Review of atrophic gastritis and intestinal metaplasia as a premalignant lesion of gastric cancer. J Cancer Prev. 2015;20(1):2540.

30. Chey WD, Wong BC. American college of gastroenterology guideline on the management of helicobacter pylori infection. Am J Gastroenterol. 2007;102(8):1808-1825.

31. Redéen S, Petersson F, Törnkrantz E, et al. Reliability of diagnostic tests for helicobacter pylori infection. Gastroenterol Res Pract. 2011:6. 